\title{
La Gestión Turística basada en el Sumak Kawsay hacia la felicidad local
}

\section{Tourism Management based on Sumak Kawsay towards local happiness}

Jenny Elizabeth Suárez Velasco. ${ }^{1}$, Gustavo Vladimir Paladines. ${ }^{2}$ \& Segundo Fernando Capa Paladines. ${ }^{3}$

\section{Recibido: 16-07-2020 / Revisado: 14-08-2020 / Aceptado: 11-09-2020 / Publicado: 03-10-2020}

\begin{abstract}
.
DOI: https://doi.org/10.33262/cienciadigital.v4i4.1431

Tourism is an economic activity that has grown significantly in recent years globally, generating positive impacts on income, employment, security, peace, among others. Ecuador has also improved its tourism management during recent years, according to the increase in arrivals and awards for its natural, cultural and infrastructure attractions. Important changes have been made in the country since 2008, when the Constitution of the Republic was modified to add the concept of Sumak Kawsay, translated as Good Living, which aims among other things, the welfare of the population; part of this well-being sought, could be achieved with the happiness of the inhabitants, a theme that took force from 2012, when the UN declared March 20 as the international day of happiness. The study sought to find out the current situation of tourism, the policies of the Sumak Kawsay since 2008 and the happiness rates that the country has. For this, the information was researched in documentary sources and by means of the synthetic analytical method; relations were established between the 3 objectives. Undoubtedly, tourism generates happiness for those who travel, but in the country, there has been no clear relationship between tourism and local happiness, because, although tourism management increased significantly, the position occupied by Ecuador is similar to that in 2013 .
\end{abstract}

\footnotetext{
${ }^{1}$ Universidad de las Fuerzas Armadas-ESPE, Escuela de Turismo, Sangolquí, Ecuador, jesuarez1@espe.edu.ec

${ }^{2}$ Universidad de las Fuerzas Armadas-ESPE, Escuela de Turismo, Sangolquí, Ecuador, gvpaladines@espe.edu.ec

${ }^{3}$ Universidad Internacional del Ecuador-UIDE-LOJA, Graduado de la Escuela de Gestión Turística y Medioambiente, Loja, Ecuador, csegundofernando@yahoo.es
} 
Keywords: Ecuador, Tourism, Social policy, National policy, Social welfare, Work welfare.

\section{Resumen.}

El turismo es una actividad económica que ha crecido de manera significativa durante los últimos años a nivel global, generando impactos positivos en ingresos, empleo, seguridad, paz, entre otros. Ecuador también ha mejorado en su gestión turística durante los últimos años, según incremento de llegadas y premiaciones por sus atractivos naturales, culturales e infraestructura. En el país se han efectuado cambios importantes desde 2008, cuando se modificó la Constitución de la República para añadir el concepto del Sumak Kawsay, traducido como Buen Vivir, el que pretende entre otras cosas, el bienestar de la población; parte de este bienestar buscado, se podría lograr con la felicidad de los habitantes, tema que tomó fuerza desde 2012, cuando la ONU declaró al 20 de marzo como el día internacional de la felicidad. El estudio procuró averiguar la situación actual del turismo, las políticas del Sumak Kawsay desde 2008 y los índices de felicidad que tiene el país. Para esto se investigó la información en fuentes documentales y por medio del método analítico sintético se establecieron relaciones entre los 3 objetivos. Es indudable que el turismo genere felicidad para quien viaja, pero en el país no ha existido una clara relación entre turismo y felicidad local, debido a que, si bien la gestión turística aumentó significativamente, el puesto que ocupa Ecuador es parecido al que tenía en 2013.

Palabras claves: Gestión turística, Felicidad, Sumak Kawsay.

\section{Introducción.}

El turismo constituye en la actualidad una de las actividades que registra un importante crecimiento a nivel global; lo cual produce ingresos significativos para las economías de los diferentes países. Es así que, según datos de la Organización Mundial de Turismo, esta actividad ha generado un 10\% del PIB mundial, así también una de cada diez personas ocupadas trabaja en actividades relacionadas con turismo. Además, acarrea otros beneficios como por ejemplo la preservación cultural, la protección del medio ambiente, el fortalecimiento de la paz, entre otros. (OMT, 2018)

A nivel mundial, el turismo se incrementó entre 2005 y 2017 en un 66\%, de acuerdo a datos del Banco Mundial (2019). Por esto, la actividad turística es sin duda una de las más representativas, no solo por el movimiento de personas que impacta en distintos sectores de las economías y por tanto en el PIB mundial, sino también porque de esta manera aporta a la generación de alegría y paz en los viajeros.

En cuanto al turismo en el Ecuador, entre 2000 y 2018 la llegada de turistas del exterior ha tenido un incremento de casi 3 veces (MINTUR, 2018). Esto muestra que en el país la 
actividad ha experimentado un impacto mayor que el promedio mundial, provocando consecuencias como el incremento de zonas turísticas, recuperación de lugares atractivos, generación de plazas de empleo, medidas de seguridad en distintas ciudades, cuidado del ambiente, refuerzo de las raíces culturales, etc.

El aumento turístico del país no solo se refleja en la cantidad de personas que llegan a visitarlo, o por los ingresos que estos turistas dejan en el territorio nacional, sino también por la cantidad de turismo interno que se fortalece cada año. En este sentido, la Ley de Feriados Nacionales y Locales de Ecuador (Asamblea Nacional, 2016), ha generado un impacto positivo de cara al turismo, debido a que permite que ciertos días festivos o de celebración se trasladen a los días lunes o viernes, los que, al juntarse con el fin de semana, generan más oportunidades para que los ecuatorianos puedan conocer los diferentes rincones de su patria, ya sea que estén cerca o lejos de su lugar de residencia.

La actividad turística está en constante evolución, ya no es el viaje por motivos de trabajo o visita, sino más bien se ha convertido en una atracción por diversos motivos u objetivos. Al respecto, Quesada (2006), afirma que existe una gran variedad de tipos de turismo, entre los que resaltan por ejemplo el cultural, deportivo, de placer, de salud, naturalista, de aventura, religioso, rural, agroturismo, etc.

En esta evolución del turismo, se puede destacar que dicha actividad tiene una relación con la felicidad. Para el Ministro de Turismo de Korea, Kim Jongdeok, la actividad turística representa sin duda una actividad que apoya al logro de la ansiada felicidad para quienes visitan diferentes destinos, afirmación que se efectuó en el Foro de Turismo Cultural del año 2014, en el que también se resaltó que "En todo el mundo la gente disfruta de viajar" (Han \& Sojung, 2014).

Si bien la actividad turística causa bienestar en quien visita un destino, que pasa con la felicidad local de quienes proveen servicios de alojamiento, traslado o alimentación al turista. Al momento no se ha encontrado estudios que traten exclusivamente este asunto; sin embargo, hay una investigación de Charaja y Mamani (2014), que se enfocó en la satisfacción laboral y motivación en una entidad regional de turismo de Perú, en la que llegaron a la conclusión de que las personas sentían más satisfacción y motivación respecto de factores internos que de los externos, entre los que contaban aspectos como la normativa legal, seguridad, entre otros.

Otra de las dudas que existen alrededor de la inquietud anterior, partiendo de uno de los aspectos que no generaron motivación en los trabajadores de la entidad peruana, es qué debe hacer el Estado para que el turismo genere felicidad local. Al respecto, esta investigación pretende analizar información estadística respecto del turismo, políticas nacionales y la felicidad local en Ecuador, para tratar de generar reflexiones que se constituyan en un primer acercamiento a este importante tema. 
Desde hace unos años se ha comenzado a tratar de medir la felicidad en los diferentes países; de acuerdo al primer Informe Mundial de la Felicidad realizado por Helliwell, Layard y Sachs (2012), en el mundo se vive una contradicción ya que, a pesar de los avances tecnológicos actuales, existe una parte significativa de la población que no tiene el alimento cotidiano necesario, se destruye el entorno natural y existen males de la vida moderna como diabetes, depresión, etc. Por esto, la vida material debería satisfacer las necesidades humanas, entre las que cuenta el alcanzar la felicidad.

En el informe citado también se destaca que la felicidad no es proporcional al ingreso de las personas, lo que se ha podido evidenciar en países como Estados Unidos de Norte América en los que a pesar del incremento del ingreso per cápita, no hubo una mejora de igual magnitud en su indicador de felicidad a nivel de sociedad.

Así también, Helliwell, Layard y Sachs (2012) señalan que una gran parte de las personas alrededor del mundo está de acuerdo con el fomento de la felicidad en todas las sociedades. Pero la visión tradicional ha sido que la felicidad es algo subjetivo que cada individuo debe buscar por sí mismo en esta vida, y por ser subjetiva no podría incluirse entre los objetivos estatales. Esta creencia está dando un giro rápido, y al aplicar el estudio de la felicidad a diferentes países del mundo, se comprobó que es posible consultar a los individuos que tan felices o satisfechos se sienten con sus vidas, lo cual proporciona información relevante no solo para cada persona, sino también para que un gobierno se base en estos resultados y tome acciones concretas en las políticas públicas.

Relacionando el tema de felicidad con el estado, para el caso del Ecuador, hay que partir del hecho de que en 2008 se modificó la Constitución de la República, a raíz de la que se han establecido algunos lineamientos basados en el concepto del "Sumak Kawsay", o traducido de alguna manera como el "Buen Vivir", que se cita en el preámbulo de dicha carta magna como: "Una nueva forma de convivencia ciudadana, en diversidad y armonía con la naturaleza, para alcanzar el buen vivir, el sumak kawsay..." (Asamblea Constituyente, 2008)

Como aspectos relevantes de la Constitución de la República del Ecuador, se resalta el término "buen Vivir" en muchos de sus artículos. De hecho, en el numeral 5 del artículo 3 de este documento, se afirma que el Estado debe efectuar la planificación para asegurar el Buen Vivir; mientras que en el artículo 74 se afirma que "Las personas, comunidades, pueblos y nacionalidades tendrán derecho a beneficiarse del ambiente y de las riquezas naturales que les permitan el buen vivir"; lo que hace alusión a lo que es el turismo como una actividad para generación recursos y por tanto apoye a la felicidad. (Asamblea Constituyente, 2008)

Con el fin de comprender los principales conceptos utilizados en este estudio, a continuación, se hace una breve referencia a sus definiciones consultadas.

Para entender el impacto de la Gestión Turística, se buscó el término gestión, que según la Real Academia Española presenta 3 definiciones: "Llevar adelante una iniciativa o un 
proyecto"; "Ocuparse de la administración, organización y funcionamiento de una empresa, actividad económica u organismo"; y finalmente, "Manejar o conducir una situación problemática" (RAE, 2018). Estas definiciones permiten validar a la gestión como el hecho de administrar y efectuar acciones encaminadas al manejo de una actividad sea empresarial o de otra índole.

En cuanto al turismo, la definición proporcionada por la Organización Mundial de Turismo en la conferencia de Ottawa en el año de 1993 fue que constituye un: “...conjunto de actividades que realizan las personas durante sus viajes y estancias en lugares distintos al de su entorno habitual, por un período de tiempo consecutivo inferior a un año con fines de ocio, por negocios y otros motivos.” (Guzmán \& García, 2015)

Al revisar el significado de la palabra turismo, por un lado, se entiende que vino de la palabra inglesa "tour", que de acuerdo al Diccionario de Oxford podría definirse como "placer de viajar a través de un país o pueblo, visitando lugares o cosas de interés". Por otro lado, turismo proviene de la palabra en idioma francés "tour" que provino del latín: "torn us" y "torn are" que significan "lo que da vueltas" y "redondear o girar" respectivamente; con lo cual el significado podría ser algo así como dar vueltas o pasear por diferentes lugares. (Mantecón, 2008, pág. 48)

Entonces la gestión turística puede ser aquella actividad que se efectúa para llevar a cabo aquellas acciones enfocadas en el brindar los productos y servicios, para las personas que provienen desde otros lugares diferentes, con fines recreativos, culturales, religiosos, entre otros.

Existe en el mundo una gran variedad de tipos de turismo, que corresponden a los motivos u objetivos que tienen los diferentes viajeros. En Ecuador se ofrecen algunos tipos de turismo como son: Deporte y Aventura, Arqueológico, Cultural, Salud, Ecológico, Vacacional, Idiomático, Comunitario y Agroturismo, de los que se pueden hallar distintas opciones en las 4 regiones con las que cuenta el país. (DiscoverEcuadorAndMore, 2017)

De estos, como señala Rosillo (2017), resaltan el de aventura que incluye actividades variadas como rafting, snorkel, puenting, canoping, entre otras; así como el sustentable o comunitario que es el que permite que los turistas convivan con las personas del destino, compartiendo sus tradiciones e incluso rutinas cotidianas.

Por otro lado, en cuanto al término felicidad, Bormans (2014) señala que, si bien puede ser utilizado de diferentes maneras, hace referencia a los que es bueno, pero de manera concreta podría entenderse "...como el grado en el cual un individuo juzga su calidad de vida en general de una manera favorable. En otras palabras: qué tanto le gusta a uno la vida que lleva.” (pág. 338) 
Desde otra perspectiva, Cutler (2014), afirma que la felicidad no puede confundirse con placer, y que, si bien existe la necesidad de buscar la satisfacción de las necesidades corporales, el camino a la felicidad tiene más relación con la mente que con el corazón; es decir, hay que entender a la felicidad desde una perspectiva menos material.

Los autores del Informe Mundial de la Felicidad, Helliwell, Layard y Sachs (2013), definen a la felicidad como "... una aspiración de todo ser humano, y también puede ser una medida del progreso social". Esto en virtud de la medición que han efectuado en distintos países del mundo. Los autores señalan “...que la felicidad se usa al menos de dos maneras: la primera como emoción... Y la segunda como una evaluación.” (pág. 3)

Para el Informe de la Felicidad 2019, Helliwell, Layard y Sachs (2019) hacen un análisis de la felicidad y el gobierno, en el que señalan que existe una relación directa de las acciones que toman los gobernantes en los niveles de felicidad de las personas; pero también existe una incidencia de las personas felices respecto de qué tipo de gobierno.

Finalmente, la medición que los autores Helliwell, Layard y Sachs (2013), llevaron a cabo para establecer el ranking de la felicidad, se basó en 6 elementos que son: PIB per cápita, apoyo social, esperanza de vida saludable, libertad para tomar decisiones de vida, generosidad y percepciones de corrupción (pág. 9). En el presente estudio se considera el ranking de los autores citados desde 2012 hasta el último efectuado en 2019, como base para revisión de los niveles de felicidad de Ecuador.

En el Ecuador se ha escuchado desde hace algunos años el término "Sumak Kawsay", el cual se mencionó de manera formal en la Constitución de la República del Ecuador de 2008, y se lo traduce como el "Buen vivir". Sin embargo, Alcántara, Garía y Sánchez (2018), analizan el origen de las palabras en idioma quichua: el significado de Sumak hace referencia a “...plenitud, grandeza, lo justo, completamente, lo superior”; mientras que Kawsay equivale a "...vida en realización permanente, dinámica y cambiante". Al unirse estas palabras, la definición sería "estado de plenitud de toda la comunidad vital". (pág. 206)

Por su parte Manosalvas (2014), señala que la traducción de Sumak Kawsay no es correcta, porque Sumak no equivale a bueno, sino que más bien el término quichua Allí equivale a bueno, y el Buen Vivir debería ser en esa lengua nativa ecuatoriana Allí Kawsay, en lugar de Sumak Kawsay como se lo usa regularmente. (pág. 114)

Lo importante del concepto ancestral andino es la finalidad como lo explica Ramírez (2008):

...la consecución del florecimiento de todos y todas, en paz y armonía con la naturaleza, para la prolongación indefinida de las culturas humanas. El Buen Vivir implica que las libertades, oportunidades, capacidades y potencialidades reales de los individuos se amplíen y florezcan de modo que permitan lograr simultáneamente aquello que la sociedad, los territorios, las diversas identidades colectivas y cada uno — visto como un ser humano universal y particular 
a la vez - valora como objetivo de vida deseable (tanto material como subjetivamente, y sin producir ningún tipo de dominación a un otro). (pág. 10)

Finalmente, se puede entender a la felicidad desde la perspectiva de la economía de consumo, y en ese sentido, ese término podría intentar garantizar al capitalismo. Por su parte, el Buen Vivir representa un planteamiento distinto de desarrollo, que se enfoca desde una manera distinta de cómo se ve y se vive la vida entendida desde una armonía con naturaleza, comunidad, mercado y Estado. Pero "La felicidad sí puede ser un medio para alcanzar el BV", siempre que se tome como "satisfacción de derechos, igual acceso de oportunidades y condiciones compartidas para el desarrollo". (El Telégrafo, 2013)

\section{Metodología.}

El presente estudio representa un esfuerzo para analizar lo que el estado ecuatoriano ha podido efectuar en los últimos años respecto de la gestión turística, con el fin de conseguir la felicidad local, lo cual conlleva una relevancia para el país desde algunos objetivos como, por ejemplo, el hecho de verificar que políticas y normativas ha propuesto el gobierno para mejorar la gestión turística. Otro de los objetivos de este estudio es el averiguar, según información estadística, que tan feliz es la gente en el país y cómo se relaciona este nivel de bienestar con la gestión turística que se ha realizado durante los últimos años.

Este es un primer acercamiento que se efectúa a un tema tan reciente, pero de mucho valor como lo es el de la felicidad, que tomó fuerza a partir de la declaración de la Organización de la Naciones Unidas, del 20 de marzo como el día internacional de la felicidad, a través de su resolución 66/281. Como parte de los antecedentes de la ONU para efectuar esta declaración, consta el de “....reconocer la relevancia de la felicidad y el bienestar como aspiraciones universales de los seres humanos y la importancia de su inclusión en las políticas de gobierno". (ONU, 2012)

En razón de lo explicado, el presente estudio se caracteriza por haber aplicado una investigación de tipo documental (Bernal, 2010), ya que se basó en la revisión de varios tipos de documentos para poder obtener la data requerida, entre la que se cuenta estadísticas actuales del tema abordado sobre turismo, políticas públicas referentes a la gestión turística y la felicidad en el país.

La estadística descriptiva constituyó una importante herramienta para la recopilación de la información que se requería, y poder entender que ha pasado con el turismo y el ranking de felicidad de los últimos años en Ecuador. (García, 2011)

En cuanto a los métodos utilizados en este estudio, el que destaca fue el analítico-sintético, ya que una vez que se obtuvo la información, se descompuso en partes para entenderlas y luego poder unirlas para obtener una comprensión general del tema abordado sobre el 
impacto de las políticas públicas en la gestión turística del país para alcanzar la felicidad local. (Bernal, 2010)

La información fue tomada de distintas fuentes nacionales e internacionales como por ejemplo: diarios de circulación nacional, artículos de revistas especializadas e indexadas, libros de texto, Ministerio de Turismo, Secretaría Nacional de Planificación y Desarrollo (SENPLADES), Organización Mundial de Turismo (OMT), Banco Mundial (BM), Organización de las Naciones Unidas (ONU), etc.

\section{Análisis de los Resultados.}

\section{Turismo de Ecuador en los últimos años.}

El crecimiento del turismo en el país, reflejado en base a los arribos, fue de $87 \%$ en los 12 años; mientras que el crecimiento mundial durante ese mismo período fue del $66 \%$. La línea celeste en la gráfica precedente corresponde a la tendencia de la variación de los datos nacionales de llegadas, la que destaca una pendiente moderadamente creciente, pero que en todo caso ha sido positiva para la situación del país y sobre todo algo superior a lo que ha sido el incremento global. Las llegadas del Ecuador representaron un 0,11\% del total mundial en 2005 , llegaron a representar un $0,13 \%$ en 2014 y 2015, para luego establecerse en $0,12 \%$ en el último año de los datos analizados.

Gráfico 1. Llegadas en millones de personas

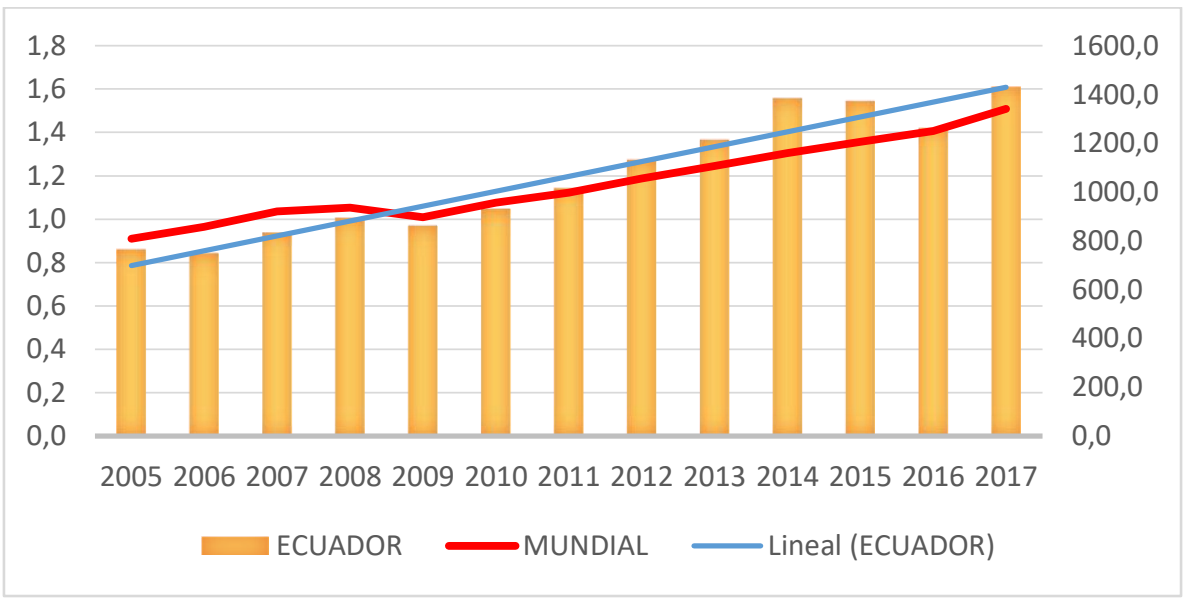

Fuente: (Banco Mundial, 2019)

Los principales 5 países de origen de quienes llegan al país desde el exterior en los últimos años han sido: Colombia, Estados Unidos, Perú, España y Venezuela. Este último destaca de una manera importante en los últimos años, pero no necesariamente por turismo, sino por migración o paso de muchos venezolanos. El gráfico 2 permite verificar las llegadas al Ecuador en color azul, y en naranja los arribos sin contar a la cantidad de venezolanos, que 
para el año 2018 fue del 39\% del total. Sin los datos de Venezuela, el crecimiento de turismo en Ecuador entre 2005 y 2017 pasaría de 87\% al 57\%, lo que implica un porcentaje inferior a la situación mundial.

Gráfico 2. Llegadas al Ecuador con y sin venezolanos

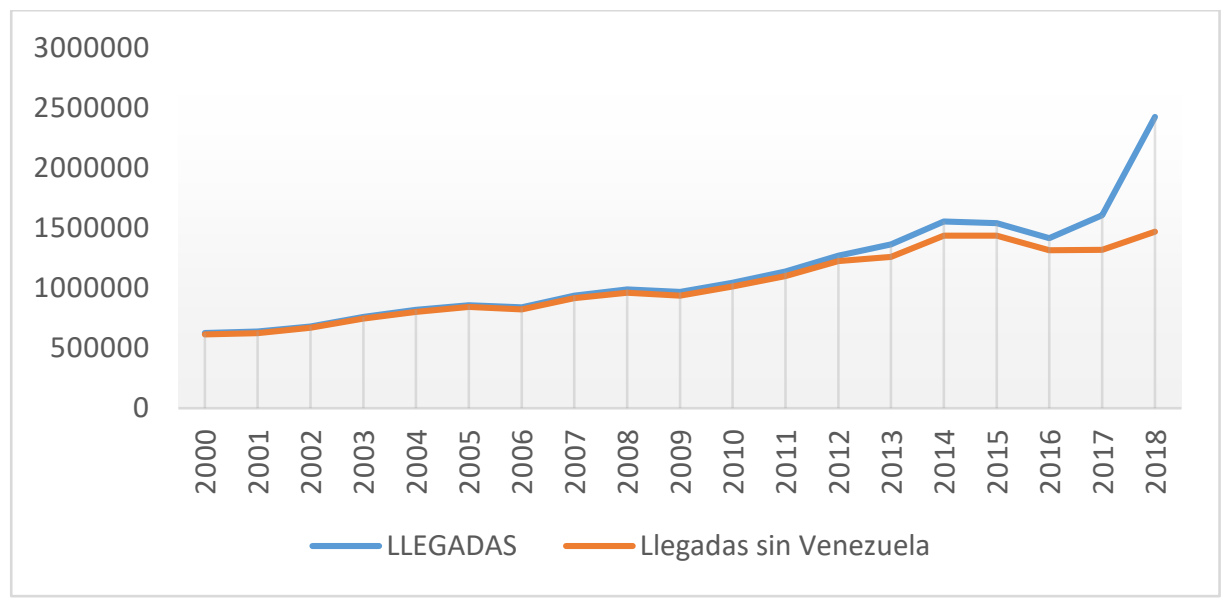

Fuente: (MINTUR, 2018)

La gráfica 3 muestra el desempeño del Producto Interno Bruto que tiene incidencia directa en el turismo del país, así como también el Valor Agregado Bruto directo de turismo. Los dos indicadores arrojan cifras de crecimiento cercanas al 40\% entre 2007 y 2016, lo que al compararlo con las cifras de crecimiento de llegadas al país sin venezolanos en ese mismo período (44\%), es inferior en 4 puntos porcentuales. En otras palabras, el incremento del ingreso que tiene relación con el turismo del país en el período analizado, ha sido levemente menor que el crecimiento de llegada de turistas.

Gráfico 3. PIB y VAB del sector Turismo en millones de USD

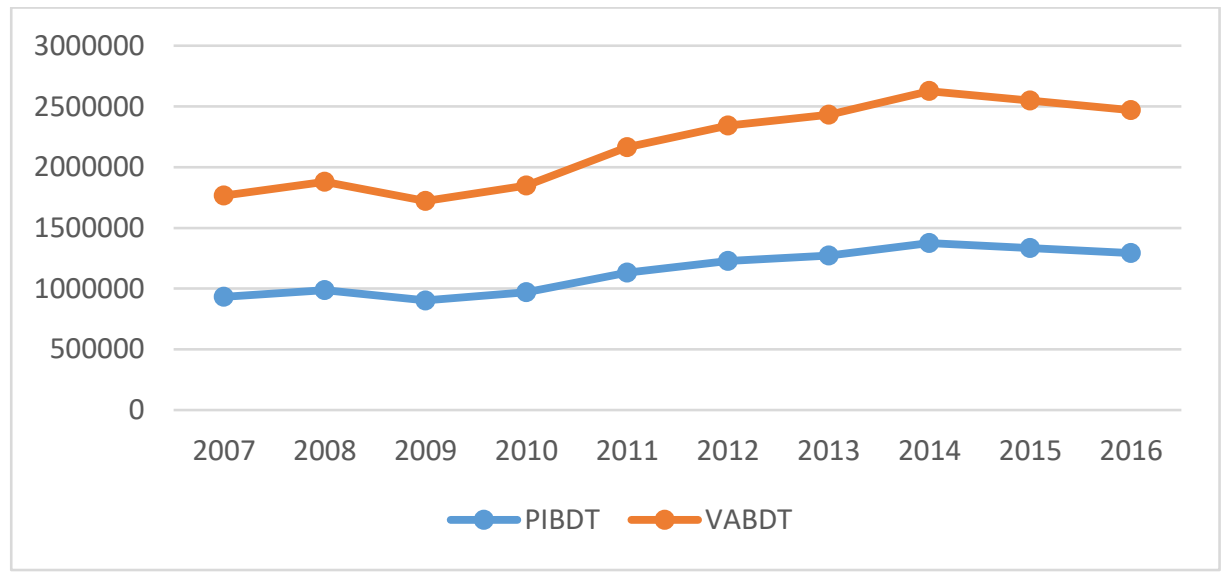

Fuente: (MINTUR, 2018) 


\section{Atractivos Turísticos de Ecuador.}

Ecuador tiene un alto potencial turístico en el mundo, gracias a sus 4 regiones claramente definidas y muy cercanas, en las que se pueden encontrar ambientes de costa, sierra, oriente e incluso a una hora las afamadas Islas Galápagos. De hecho, según información publicada por el Ministerio de Turismo, en el año 2017 se declararon como principales destinos a Quito, Galápagos, Otavalo, Guayaquil, Tren crucero, Cuenca, Parque nacional Cotopaxi, Tena, Mindo, y otros 9 destinos renqueados como los favoritos (MINTUR, 2017).

Por sus atractivos naturales, arquitectura colonial e infraestructura hotelera, Ecuador ha participado en 15 ocasiones para los premios Wold Travel Awards, y hasta 2017 recibió 131 premiaciones por diferentes motivos en distintos destinos. Por ejemplo, en 2004 obtuvo 3, en 2007 logró 9, en 2014 fueron 15 y 24 para el año 2017. Estas cifras indudablemente han permitido crecimiento turístico por la llegada de visitantes de varias partes del mundo (Encalada, 2018). Para 2018, el país recibió 3 premiso "Oscar" de la premiación Wold Travel Awards realizada en Portugal, entre los que Ecuador se destacó como "Destino Verde Líder del Mundo" (El Comercio, 2018).

Dentro del país, otro destino que resalta es su ciudad capital Quito, la que en el año 2018 obtuvo por sexta vez consecutiva el reconocimiento como "destino líder de Sudamérica". En esta última jornada de los Wold Travel Awards, el país recibió un total de 25 galardones en 38 categorías. (Jaramillo, 2018)

\section{Turismo y Empleo.}

En cuanto al empleo que genera el turismo, de acuerdo a datos del Ministerio de Turismo, el país en marzo del 2015 tuvo 405.820 personas que laboraban en esa actividad de forma directa o indirecta, de las que $67 \%$ fueron mujeres. En ese mismo año se registraba un total de 580 servidores que laboraban en la dependencia pública de turismo a nivel nacional. (MINTUR, 2015)

Pero la cifra de empleo se incrementó para los años siguientes, es así que fue de 491.698 personas para inicios del año 2018, lo que representó un 5,1\% más que el año inmediato anterior. Esto sin duda muestra que la actividad turística aporta a la generación de las plazas de trabajo en el país, ya que para 2018 el 6,3\% de ocupados correspondió a este sector. (El Telégrafo, 2018)

\section{Planificación del Ecuador en los últimos años.}

A partir del año 2007 que inició el expresidente Correa el poder ejecutivo del país, iniciaron una serie de cambios importantes para Ecuador. Entre estos, se cambió el enfoque de algunas funciones del Estado, se modificó la Constitución de la República en 2008, entre otros. Como 
parte de estos cambios, se diseñaron planificaciones plurianuales en el país, que se basaban en el concepto principal del Ecuador a partir de esa época: El Buen Vivir.

Una de las primeras planificaciones fue la denominada "Construcción de un Estado democrático para el Buen Vivir” que se elaboró para cubrir el período 2007-2012. Entre sus conclusiones, Mayorga (2007), menciona que se planteaban 3 estrategias generales; la primera fue el evitar que los grupos de poder tomen el mando de cuestiones políticas para asegurar la democracia; la segunda buscaba el transformar el estado para constituirlo "...como fuerza integradora de la sociedad"; mientras que la tercera se enfocó en fortalecer la institucionalidad político-administrativa. Todo esto con miras a alcanzar el Buen Vivir en la sociedad, para lograr el bienestar del país y sus habitantes.

Una segunda planificación fue la denominada "Plan Nacional para el Buen Vivir", en el que el señor secretario Ramírez (2009), planteaba principalmente 12 estrategias y objetivos para cumplirlos entre 2009 y 2013. En este documento se incluye como décima estrategia el "fomento al turismo comunitario", como una manera de lograr la participación de los grupos minoritarios en la actividad turística, a la vez que se debía cuidar los bienes naturales y patrimoniales. Como objetivo se destaca el 11.11.1 que se refería a "Alcanzar el 15,1\% de participación del turismo en las exportaciones de bienes y servicios no petroleros al 2013".

Luego se elaboró el "Plan Nacional Buen Vivir período 2013 al 2017", último año del ex presidente Correa, en el que planteó 12 objetivos, entre los que resalta el tercero que trata de mejorar la calidad de vida de los ecuatorianos y el décimo que pretendió "transformar la matriz productiva". En este último objetivo se planteó la meta 10.8 que buscaba incrementar a $64 \%$ el ingreso que generaba la actividad turística sobre las exportaciones. Con respecto a la felicidad, este documento se enfocaba desde la premisa de que "El Buen Vivir es la forma de vida que permite la felicidad", con lo cual el gobierno relacionó los dos conceptos. (Muñoz, 2014)

A partir de 2017 se presentó una nueva planificación denominada "Toda una Vida" en la presidencia de Lenin Moreno, en la que el secretario Mideros (2017), propone el logro de 9 objetivos en distintos ejes sobre derechos, economía y mejora del estado. En este documento se estableció la política 9.4 que busca fomentar el turismo para generar ingresos y trabajo; como meta para 2021 se fija la cifra de 2,5 mil millones de USD. En cuanto a la felicidad, este plan incluye el primer objetivo sobre garantizar una vida digna de las personas, con el fin de lograr su felicidad.

Finalmente, en 2017 el Ministerio de Turismo generó un documento de Política de Turismo, en la que se destaca la importancia de esta actividad económica, y se buscan objetivos concretos de mejora en cuanto a competitividad, PIB, empleo e inversión para el año 2030, con lo que se pretende convertir al país "...en un referente turístico de la región..." (MINTUR, 2017) 


\section{Gasto en promoción turística.}

De acuerdo a cifras publicadas por el Ministerio de Turismo, el gasto de promoción turística que ha realizado el gobierno en los últimos años ha tenido un crecimiento del $92 \%$ entre 2007 y 2016. Como se observa en la gráfica siguiente, el gasto no tiene una tendencia sostenida, sino que en cada período varía, siendo el 2014 en el que se destinó una mayor cifra (48.170 miles de USD), y el año 2008 en donde el valor fue de apenas 2.043 miles de USD. La relación porcentual de cada año también es muy variada, pero a excepción de 2008, se ha mantenido sobre el 40\%, lo cual implica que el Estado si invierte en la promoción turística del país, como medida de apoyo a la generación de mayores ingresos de turistas extranjeros.

Gráfico 4. Gasto en promoción turística en miles de USD

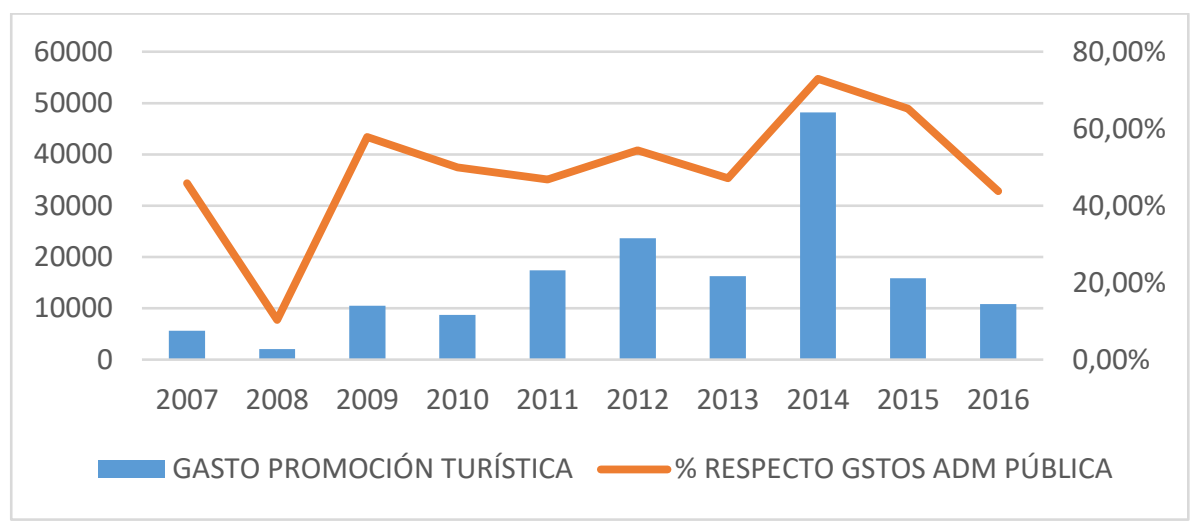

Fuente: (MINTUR, 2017)

\section{Ranking de felicidad de Ecuador entre 2012 y 2017}

De acuerdo a los Informes de la felicidad Ecuador a mejorado su puesto en el ranking, como se muestra a continuación:

Gráfico 5. Ranking de felicidad del Ecuador

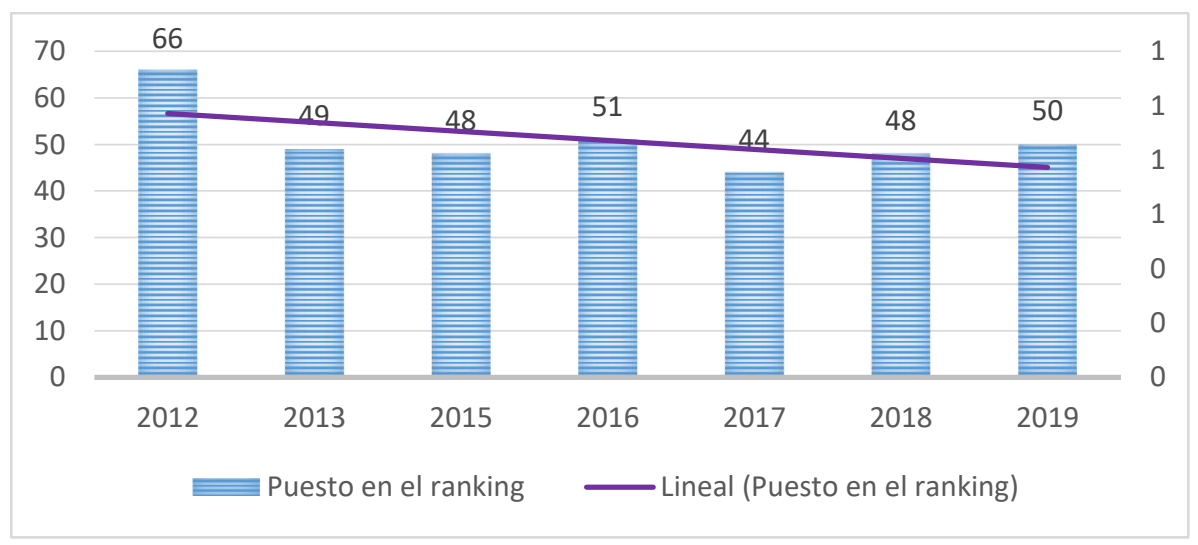

Fuentes: (Datos Macro, 2019), (Helliwell, Layard, \& Sachs, 2012) 
Si se toma en consideración el primer informe del año 2012, hasta 2019 el país mejoró 24\%, ya que disminuyó 16 posiciones; sin embargo. La mejor posición para Ecuador fue en 2017 (44) y desde allí a seguido bajando 6 puestos en dos años, lo que de alguna manera indica que la situación de bienestar a disminuido 13\% hasta 2019.

\section{Comparativo con otros dos países de la región.}

La gestión de turismo de Ecuador es mucho menor que Colombia y Chile, en los que el crecimiento de estos últimos años fue de $348 \%$ y $218 \%$ respectivamente, como lo muestra la siguiente gráfica:

Gráfico 6. Llegadas de turistas a Ecuador, Chile y Colombia en millones de personas

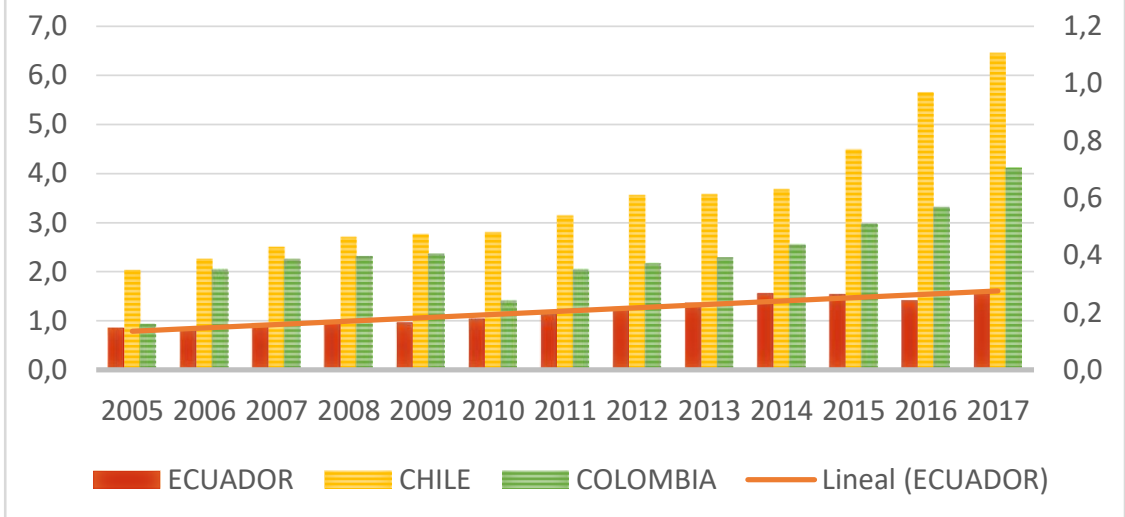

Fuentes: (Banco Mundial, 2019)

En cuanto a la felicidad, Ecuador ocupa un puesto inferior a otros países del mundo como Japón, China, Perú, etc. Sin embargo, tiene varios de su región mejores puntuados como Chile y Colombia.

Gráfico 7. Ranking de felicidad de Ecuador, Chile y Colombia

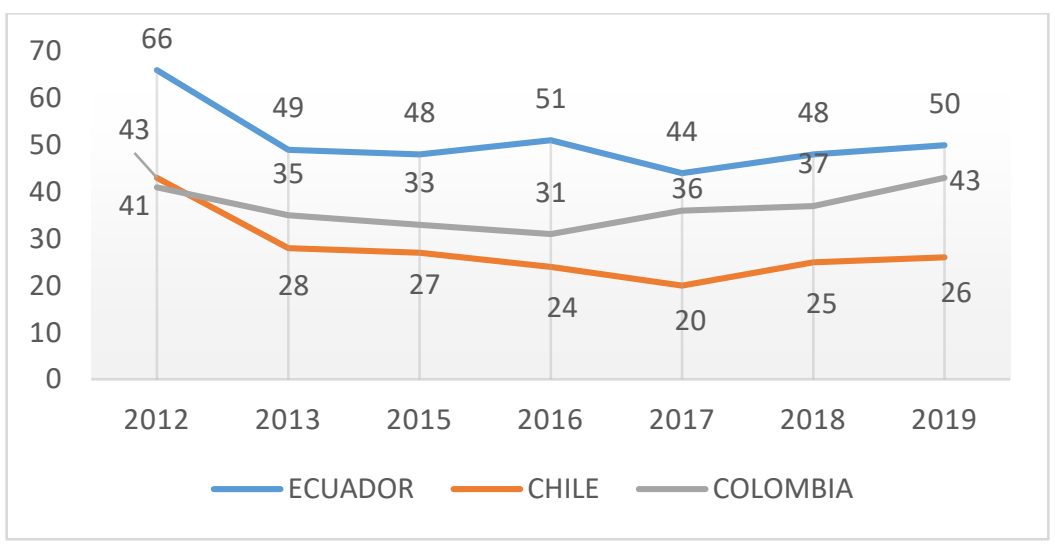

Fuentes: (Datos Macro, 2019), (Helliwell, Layard, \& Sachs, 2012) 
Chile presenta una tendencia parecida a Ecuador, pero ha logrado mantenerse en la posición 26 para 2019, lo cual indica que su gente es casi el doble más feliz que en el país analizado. En cuanto a Colombia, ha disminuido notablemente su índice de felicidad a 2019 (43), respecto de su punto mínimo en 2016 (31).

El PIB per cápita de los 3 países comparados tiene de alguna manera una relación con el puesto en el informe de la felicidad presentado en la gráfica anterior. Chile tiene presenta un crecimiento del $43 \%$ en los años analizados, mientras que Ecuador refleja un incremento del $55 \%$; sin embargo, la cifra de Chile es más del doble que Ecuador, la cual es algo inferior a la de Colombia, que solo creció $18 \%$ en estos 7 años.

Gráfico 8. PIB per cápita de Ecuador, Chile y Colombia en Euros

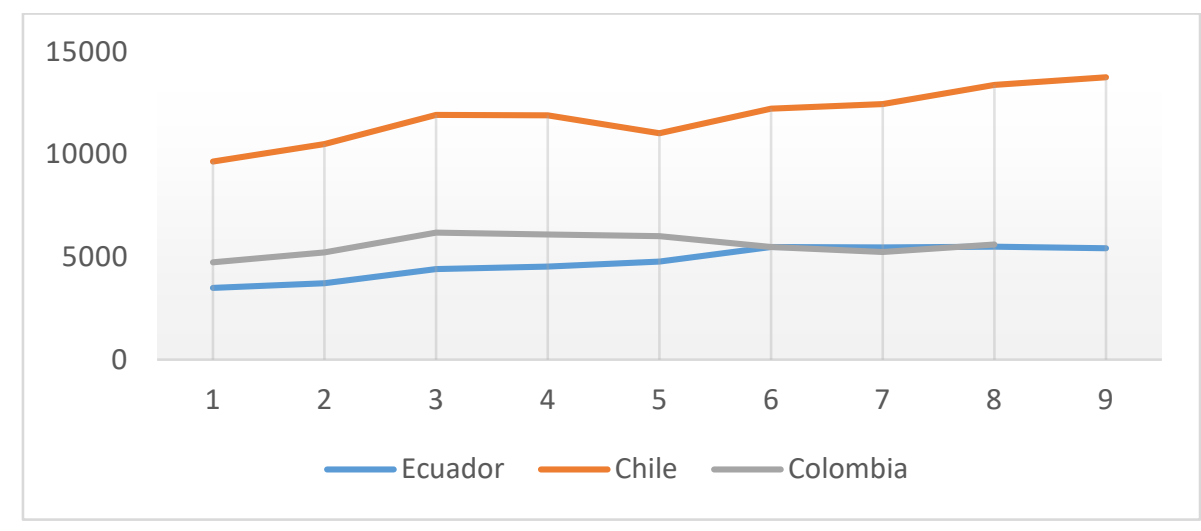

Fuentes: (Datos Macro, 2019)

\section{Conclusión.}

- Desde hace algunos años el turismo representa una actividad que ha tomado mucha importancia en el mundo. En el caso del Ecuador la situación es similar, aunque no con la misma fuerza, pero ha incrementado las llegadas de turistas; y, por tanto, los ingresos y plazas de empleo por esta actividad. Sin embargo, todavía se debe hacer mucho en cuanto al turismo, para aprovechar los virtuosos escenarios naturales con los que cuenta la patria, sus tradiciones y actividades de aventura, agroturismo, etc., que han sido reconocidos y premiados durante los últimos 14 años.

- El gobierno del Ecuador trató de iniciar un cambio importante para el país con la modificación de la Constitución de la República en 2008, en la que incluyó al "Buen Vivir" como un concepto que debe respetar los derechos de las personas, sus oportunidades y equidad, dentro de un margen de armonía con el entorno natural y cultural. Si bien han mejorado las planificaciones en referencia al turismo y a la felicidad, por parte del Estado hace falta concretar más recursos para fortalecer la actividad turística. Es contradictorio que por un lado se establezca una política de turismo con metas importantes al 2030, y por otro se asigne a esta cartera de estado para 2019 la inversión más baja desde 2011 (Encalada, 2019). 
- En cuanto a la felicidad, como sostienen Helliwell, Layard y Sachs (2019), es de vital importancia que los estados consideren al bienestar para incluirlo en su planificación, ya que las personas se centran cada vez más en este tema, que es algo que les importa mucho. En el Ecuador se ha podido observar que, si bien se han efectuado esfuerzos desde el gobierno por esquematizar planes que consideren equidad, derechos y oportunidad, como bases para lograr una situación de bienestar de sus habitantes, ha existido un retroceso de 6 puestos en el ranking de 2019 respecto a 2017.

- El turismo y la felicidad tienen una relación directa, como lo afirma Caravaca (2019), ya que toda acción que se efectúa como parte de la gestión turística está enfocada en generar felicidad del visitante. Este sería otro motivo para que Ecuador siga vendiendo su marca en el mundo entero, como destino de felicidad, que es lo que valoran las personas en la actualidad.

- La felicidad tiene una relación directa con los ingresos, tal como lo afirma Ramírez (2008), y cuando el país invierta una cifra constante y creciente hacia la gestión turística, se podrían reducir las cifras de desempleo y de empleo inadecuado, lo que impactaría en una mejora del nivel de bienestar de los ciudadanos, y por tanto alcanzar la premisa del Buen Vivir. Sin embargo, no solo es necesario basarse en los ingresos de las personas como motivo para lograr la felicidad, ya que de acuerdo al estudio de Baucells y Sarin (2007), el tener más dinero no impacta de manera proporcional al incremento del nivel de felicidad, es mejor en muchos casos tener "comida, vivienda, dormir, amistad, actividades espirituales, etc."

- Por otro lado, al observar las cifras de países cercanos de la región que, en los ingresos per cápita son mejores que los de Ecuador, el turismo genera más visitantes y los indicadores de felicidad son mejores; se ve la necesidad de fijar objetivos más ambiciosos para la gestión de turismo, como fuente de generación de ingresos, de empleo, pero también de felicidad local.

- Finalmente, se comprobó que el turismo en el país ha crecido considerablemente, y que el estado mejoró el apoyo a la gestión turística por medio de recursos y políticas; sin embargo, todavía no se tiene una relación fuerte entre turismo y felicidad local en Ecuador, ya que falta explotar los atractivos premiados de la patria, para que lleguen más extranjeros de visita y generen ingresos que muevan la economía nacional. Así también, falta apuntalar los objetivos de mejora de la calidad de vida, en miras a cumplir el Buen Vivir incluido en la Constitución de la República de 2008.

\section{Referencias bibliográficas.}

Alcántara, M., García, M., \& Sánchez, F. (2018). Movimientos sociales: Memoria del 56. Congreso Internacional de Americanistas. Salamanca: Universidad de Salamanca.

Asamblea Constituyente. (2008). Constitución de la República del Ecuador. Montecristi: Asamblea Constituyente. 
Asamblea Nacional. (2016). Ley Orgánica Reformatoria a la Ley Orgánica del Servicio Público y al Código de Trabajo. Quito: Asamble Nacional. Obtenido de Feriados Ecuador.

Banco Mundial. (2019). Turismo Internacional, número de arribos. Obtenido de Banco Mundial: https://datos.bancomundial.org/indicador/ST.INT.ARVL

Baucells, M., \& Sarin, R. (2007). Con más dinero ¿Se puede comprar más felicidad? Navarra: IESE Universidad de Navarra.

Bernal, C. (2010). Metodología de la investigación. Bogotá: PEARSON.

Bormans, L. (2014). El libro mundial de la felicidad. Monterrey: Nómada.

Caravaca, E. (20 de marzo de 2019). Felicidad y turismo. Obtenido de Andalucía Lab: https://www.andalucialab.org/blog/felicidad-y-turismo/

Charaja, Y., \& Mamani, J. (2014). SATISFACCIÓN LABORAL Y MOTIVACIÓN DE LOS TRABAJADORES DE LA DIRECCIÓN REGIONAL DE COMERCIO EXTERIOR Y TURISMO - PUNO - PERÚ 2013. Comuni@ cción, 5-13.

Cutler, H. (2014). DALAI LAMA El arte de la felicidad. Barcelona: Penguin Random House.

Datos Macro. (2019). Expansión. Obtenido de Datos Macro: https://datosmacro.expansion.com/pib/ecuador

Datos Macro. (2019). Índice mundial de la felicidad. Obtenido de Datos Macro: https://datosmacro.expansion.com/demografia/indice-felicidad/ecuador

DiscoverEcuadorAndMore. (mayo de 2017). Tipos de Turismo. Obtenido de Discover Ecuador and More: http://discoverecuadorandmore.com/tipos-de-turismo

El Comercio. (4 de diciembre de 2018). Ecuador obtuvo tres 'Oscar' en la gala mundial de los World Travel Awards. Obtenido de Diario El Comercio: https://www.elcomercio.com/tendencias/ecuador-oscar-turismo-portugalpremio.html

El Telégrafo. (28 de marzo de 2013). ¿Felicidad vs. Buen Vivir? Obtenido de Diario El telégrafo: https://www.eltelegrafo.com.ec/noticias/columnistas/1/felicidad-vs-buenvivir

El Telégrafo. (11 de junio de 2018). Empleo en turismo creció 5,1\% a inicios del año. Obtenido de Diario El Telégrafo: https://www.eltelegrafo.com.ec/noticias/economia/4/turismo-ecuador-empleoscrecimiento

Encalada, E. (14 de septiembre de 2018). Ecuador obtuvo 131 premios en 14 años en los World Travel Awards. Obtenido de Diario El Comercio: https://www.elcomercio.com/viajar/ecuador-turismo-world-travel-awards.html 
Encalada, E. (5 de febrero de 2019). Las inversiones en turismo serán las más bajas en ocho años. Obtenido de Diario El Comercio: https://www.elcomercio.com/tendencias/inversiones-turismo-ecuador-presupuestoministerio.html

García, C. (2011). Estadística descriptiva y probabilidades para ingenieros. Lima: Macro.

Guzmán, L., \& García, G. (2015). FUNDAMENTOS TEÓRICOS PARA UNA GESTIÓN TURÍSTICA DEL PATRIMONIO CULTURAL DESDE LA PERSPECTIVA DE LA AUTENTICIDAD. Obtenido de Enciclopedia Virtual EUMED: http://www.eumed.net/libros-gratis/2015/1496/turismo-cultural.htm

Han, J., \& Sojung, Y. (11 de noviembre de 2014). El turismo contribuye a alcanzar la felicidad y la paz en el mundo. Obtenido de KOREA.net: http://spanish.korea.net/NewsFocus/Policies/view?articleId=122899

Helliwell, J., Layard, R., \& Sachs, J. (2012). World Happiness Report. New York: Sustainable Development Solutions Network.

Helliwell, J., Layard, R., \& Sachs, J. (2013). World Happiness Report 2013. New York: Sustainable Development Solutions Network.

Helliwell, J., Layard, R., \& Sachs, J. (2019). World Happiness Report. New York: Sustainable Development Solutions Network.

Jaramillo, I. (18 de septiembre de 2018). Quito, por sexta vez, logró premio como destino líder de Sudamérica. Obtenido de Diario El Universo: https://www.eluniverso.com/noticias/2018/09/18/nota/6958739/quito-sexta-vezlogro-premio-como-destino-lider-sudamerica

Manosalvas, M. (2014). Buen vivir o sumak kawsay. En busca de nuevos referenciales para la acción pública en Ecuador. ÍCONOS, 101-121.

Mantecón, A. (2008). La experiencia del turismo: un estudio sociológico sobre el proceso turístico- residencial. Barcelona: Icaria.

Mayorga, G. (2007). Del Estado Burgués al Estado del Buen Vivir. En E. Flor, Construcción de un Estado democrático para el Buen Vivir: Análisis de las principales transformaciones del Estado Ecuatoriano (págs. 277-294). Quito: SENPLADES.

Mideros, A. (2017). Plan Nacional de Desarrollo 2017-2021 Toda una Vida. Quito: SENPLADES.

MINTUR. (30 de abril de 2015). Turismo genera en Ecuador 405.820 mil fuentes de trabajo. Obtenido de Ministerio de Turismo: https://www.turismo.gob.ec/turismo-genera-enecuador-405-820-mil-fuentes-de-trabajo/

MINTUR. (2017). De acuerdo a información publicada por el Ministerio de Turismo, en Ecuador existen . Quito: Dirección de Productos y Destinos del Ministerio de Turismo de Ecuador. 
MINTUR. (2017). Política de Turismo del Ecuador. Quito: Ministerio de Turismo.

MINTUR. (2017). Promoción Turística. Obtenido de Ministerio de Turismo: https://servicios.turismo.gob.ec/index.php/18-turismo-en-cifras/analisiseconomico/97-cuenta-satelite-de-turismo

MINTUR. (19 de septiembre de 2018). Moviemientos Internacionales. Obtenido de Ministerio de Turismo Ecuador: https://servicios.turismo.gob.ec/index.php/turismocifras/2018-09-19-17-01-51/movimientos-internacionales

Muñoz, P. (2014). Plan Nacional Buen Vivir 2013-2017. Quito: SENPLADES.

OMT. (junio de 2018). La importancia del Turismo. Obtenido de Organización Mundial de Turismo: http://media.unwto.org/content/infographics

ONU. (2012). Día Internacional de la Felicidad, 20 de marzo. Obtenido de Organización de las Naciones Unidas: https://www.un.org/es/events/happinessday/background.shtml

Quesada, R. (2006). Elementos del Turismo. San José: EUNED.

RAE. (2018). Diccionario de la lengua española. Obtenido de Real Academia Española: https://dle.rae.es/?id=JAQijnd

Ramírez, R. (2008). La felicidad como medida del Buen Vivir en Ecuador. Entre la materialidad y la subjetividad. Quito: SENPLADES.

Ramírez, R. (2009). Plan Nacional para el Buen Vivir 2009-2013. Quito: SENPLADES.

Rosillo, I. (21 de diciembre de 2017). Rentabilidad económica del turismo en el Ecuador. Obtenido de Blog de la Universidad Técnica Particular de Loja: https://noticias.utpl.edu.ec/rentabilidad-economica-del-turismo-en-el-ecuador.

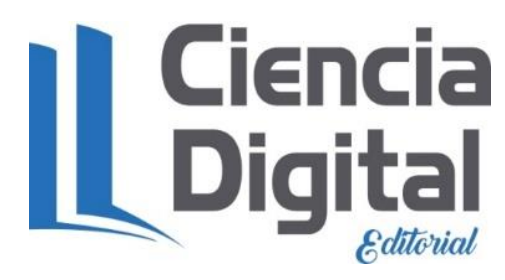


PARA CITAR EL ARTÍCULO INDEXADO.

Suárez Velasco, J. E., Paladines, G. V., \& Capa Paladines, S. F. (2020). La Gestión Turística basada en el Sumak Kawsay hacia la felicidad local. Ciencia Digital, 4(4), 85-103. https://doi.org/10.33262/cienciadigital.v4i4.1431

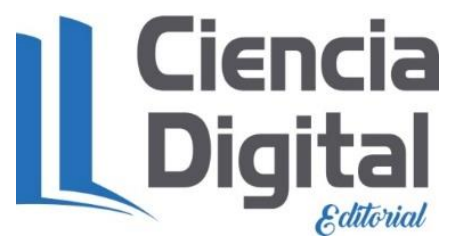

El artículo que se publica es de exclusiva responsabilidad de los autores y no necesariamente reflejan el pensamiento de la Revista Ciencia Digital.

El artículo queda en propiedad de la revista y, por tanto, su publicación parcial y/o total en otro medio tiene que ser autorizado por el director de la Revista Ciencia Digital.
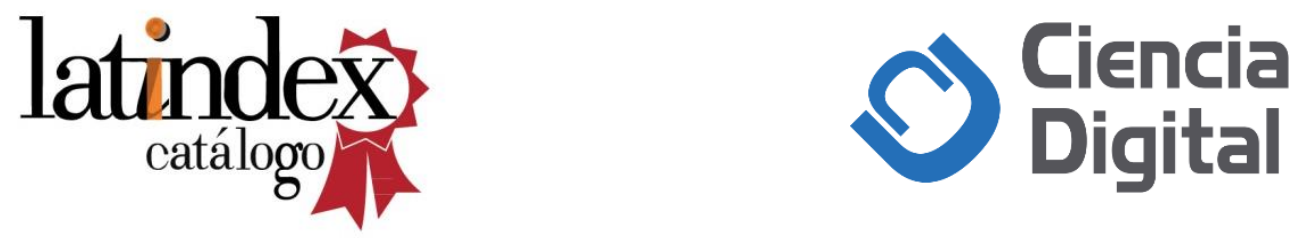